\title{
Sujeto político y vida pública: privatización de la educación en Chile y sus consecuencias en los sujetos que se educan
}

\author{
Mónica Peña Ochoa \\ Universidad Diego Portales, Santiago, Chile. \\ Email: mpenaochoa@gmail.com
}

Resumen: El artículo asume que el complejo proceso privatizador y descentralizador que vivió el sistema educativo chileno desde el año 1981 ha tenido consecuencias en el sujeto que se educa, a través de problemáticas que se concentran en los actores educativos -específicamente los estudiantes-. Estas problemáticas se han definido como la “psicologización” de los problemas del sistema educativo, la supuesta "clientelización" de los alumnos y el lugar que le cabe a La Familia como figura preponderante en el proceso privatizador. Las consecuencias de esto dicen relación con la consolidación del sistema privatizado en la forma de enfrentar las subjetividades y, muy especialmente, en el quiebre del rol público que le cabe a la escuela y a la educación.

Palabras clave: Privatización, descentralización, actor educativo, “psicologización”, “clientelización”, sujeto político

\section{Political subject and public life: privatization of education in Chile and its impact on the subjects being educated}

\begin{abstract}
The paper assumes that the complex privatization and decentralization process experienced by the Chilean education system since 1981 has had an impact on the individual who is educated, through issues that focus on those involved in education, specifically the students. These problems have been identified as the "psychologization" of the problems of the educational system, the supposed "customerizing" of students and the place that corresponds to the family as dominant figure in the privatization process. The consequences of this are related to the consolidation of the privatized system in the way of dealing with subjectivity, and especially, in the breakdown of the public role of school and education.

Key words: Privatization, decentralization, actor, “psychologization”, “customerizing”, political subject
\end{abstract}

\section{Sujeito político e vida pública: a privatização da educação no Chile e seu impacto sobre os indivíduos que são educados}

Resumo: Este artigo pressupõe que o complexo processo de descentralização e privatização vivida pelo sistema educativo chileno desde 1981 
teve consequências para o indivíduo que é educado através de questões que incidem sobre os envolvidos na educação, especificamente os alunos. Estas questões têm sido definida como a "psicologização" dos problemas do sistema educativo, o suposto "clientelização" de estudantes e o lugar que se encaixa na família como uma figura de destaque no processo de privatização. As conseqüências estão relacionadas com a consolidação do sistema privatizado na forma de lidar com a subjetividade e, particularmente, na quebra do papel público da escola e da educação.

Palavras-chave: privatização, descentralização, ator educacional, “psicologização”, “clientelização”, sujeito político

$$
* * *
$$

\section{Introducción}

La descentralización de la educación chilena, así como su consiguiente privatización, han sido tópicos ampliamente analizados por el mundo académico, así como también han sido reconocidos como el nudo problemático en la crisis educacional que el país atraviesa ${ }^{1}$. Sin embargo, hay una disyunción importante a nivel de análisis entre los cambios a nivel macro y su influencia a nivel micro. En este caso, la pregunta es acerca de la influencia que un modelo descentralizado que ha sido planificado e implementado sin la participación de la ciudadanía, ha tenido sobre los actores primarios del mundo educativo, en este caso, los niños, niñas y jóvenes que estudian en el sistema escolar chileno. Como tal empresa -la de analizar esta relación- es difícil de limitar, este artículo pretende simplemente sentar algunas bases para reflexionar acerca de este tema, que ha sido soslayado como si los cambios políticos y los cambios subjetivos fueran por carriles distintos.

El sistema institucional educativo en Chile, además, ha desincentivado la participación y la pregunta por el sujeto que se educa, como veremos, por varias razones que se relacionan todas con la desconexión que el modelo neoliberal bajo el cual se diseño el sistema educativo chileno, mantienen con las demandas colectivas, insistiendo en la potenciación del individuo cliente que busca un servicio, el educativo. Son los y las jóvenes estudiantes, que a manera de resistencia, se enfrentan a un sistema que es injusto y que no funciona ni como vértice cultural de la república pero tampoco como servicio a un cliente determinado.

La descentralización educativa ha sido sin lugar a dudas la principal reforma al modelo educativo chileno: su universalidad y sus consecuencias no tienen parangón con otra reforma ocurrida en la historia de la educación chilena. Estas consecuencias, sostenemos, no son detalles de carácter administrativo o tienen su principal correlato en la calidad educativa. Son también consecuencias que se reflejan en la forma que los actores como sujetos políticos enfrentan la educación: ya sea en la confusión por el cambio de reglas del juego "por decreto"; ya sea por el nuevo rol que les cabe, en que ya no son sólo parte del juego de la educación sino también son parte de una relación de carácter contractual muy distinta a la sostenida anteriormente. 
Para comenzar, es necesario describir brevemente cómo es este sistema, que nace en 1981 durante la dictadura militar de Augusto Pinochet, a través de tres procesos: la descentralización, la municipalización y la privatización de la educación. Asimismo, hubo un cambio profundo en el sistema de financiamiento, que de subsidio a la oferta pasó a subsidio a la demanda a través de una subvención por promedio de asistencia de los alumnos, que se entrega de la misma forma y en los mismos montos a las escuelas municipales y a las del sector particular subvencionado (Maldonado, 2003) con un cambio desde el año 2008, como se verá, con la llamada "Subvención escolar preferencial”, que se aplica cuando se considera que existen condiciones particulares de carácter deficitario en los alumnos que implicarán un aumento en la subvención recibida por el establecimiento.

La descentralización pretendía que el Estado dejara en manos de otros actores las decisiones educativas, bajo la hipótesis de que habría mayor eficiencia y que estas decisiones (pedagógicas, curriculares, entre otras) serían tomadas de manera concertada con las comunidades de base, cuidando la libertad de educación consagrada en la Constitución chilena. La municipalización por su parte fue el proceso en que se le encargó a los Municipios (gobiernos comunales) que se hicieran cargo de la educación gestionando las escuelas bajo su territorio, que antes eran administradas centralmente por el Estado a través del Ministerio de Educación. La privatización consistió en la apertura de subsidios a privados que quisieran instalar una escuela, subsidio que se entrega -lo mismo para las municipalidades- a través de la asistencia de niños y niñas a las escuelas, es decir, no depende simplemente de la inscripción de alumnos. Este modelo fue pensado por el economista de la Escuela de Chicago Milton Friedman, formador de los principales economistas chilenos que participaron en la dictadura de Pinochet.

Una característica importante de la descentralización es un supuesto énfasis en la participación de las comunidades en las decisiones de la escuela: Consejos escolares conformados por todos los miembros de la comunidad escolar participarían en la toma de decisiones. Este eje fundamental del sistema descentralizado no se ha dado a cabalidad; y por el contrario, la enorme brecha entre escuelas de ricos y de pobres se evidencian también en los niveles de participación de las familias en las decisiones educativas, donde los más pobres, al ser menos educados y tener que lidiar con más exigencias para sustentar el hogar, suelen participar muy poco en la escuela, así como tener muy bajas expectativas puestas en ella (Elacqua, 2004; Van Der Wal, 2007).

Este sistema no fue cambiado ni adaptado por los gobiernos de la Concertación, coalición de centro - izquierda posteriores a la dictadura, a pesar de que durante el año 2006, bajo el Gobierno de la socialista Michelle Bachelet, estudiantes secundarios llevaron a cabo un masivo movimiento de protesta que pedía el cambio en este sistema principalmente a través del fin de la municipalización y la revisión del sistema de subvenciones a pri- 
vados. Si bien fueron escuchados en algunos puntos -por ejemplo, en la mayor exigencia de antecedentes académicos para los privados que quisieran montar una escuela- en el resto de sus demandas, no hubo mayor respuesta.

Así, hay una fuerte diferencia entre las escuelas municipalizadas y las que son administradas por privados, siendo generalmente las primeras las de menor calidad educacional a la vez que las más "elegidas” por las familias más pobres dada su gratuidad y cercanía física. Las segundas reciben aportes privados y en algunos casos subvención del Estado, y además de esta diferencia fundamental, son escuelas que tienen la posibilidad de seleccionar a sus estudiantes y de expulsar a los que no cumplan con las exigencias del establecimiento, no así las municipalizadas, que están obligadas por ley a mantener a sus alumnos hasta el sexto año de educación básica o primaria. De tal manera, ha habido un movimiento migratorio de buenos estudiantes de educación municipalizada (o con padres con altas expectativas educativas y mejor situación económica) a las escuelas particulares - subvencionadas, así como el movimiento inverso, de estudiantes rechazados por el sistema privado que llegan a las escuelas municipalizadas, las que a la vez tienen menos recursos económicos y simbólicos (por ejemplo, se encuentran en comunas pobres y alejadas) para hacerse cargo de los alumnos que entrañan casos complejos.

Es posible entonces reconocer que la buena calidad en materia educativa que presentan las escuelas de carácter privado es resultado de este modelo de selección del alumnado, de las mejores condiciones laborales para profesores y profesoras, y otros factores que son ajenos a la entrega de un verdadero "valor agregado" a nivel educativo, que se base en factores pedagógicos o de conocimiento o saber (Romaguera y Mizala, 2000).

En síntesis, se presenta un sistema complejo, descentralizado y privatizado, poco participativo en su creación y en la implementación, que ha tomado muy poco en cuenta a los actores primarios, "excepto como sujetos de mercado (inversores o consumidores de educación, pero no como ciudadanos con derecho a la educación y a la participación” (Redondo, 2005: 2). Este estilo poco participativo, donde las demandas de carácter colectivo no tienen espacio, es el que analizaremos a continuación.

\section{Sujetos y sistema descentralizado-privatizado: descripción y análisis del problema}

Los principales problemas que el sistema educativo chileno presenta en relación con el sujeto que se educa podrían sintetizarse como sigue: a) la segmentación en estratos sociales determinados que generan expectativas y resultados de aprendizaje definidos, siempre en desmedro de los sujetos más pobres; b) la "psicologización” de los problemas educativos que la estratificación provoca, en parte consecuencia del primer punto; c) la 
consolidación de un alumno consumidor en el aula de la escuela privada, con la consecuente precarización de la relación profesor - alumno en torno del saber y el conocimiento; y finalmente, d) la privatización de la educación y su estrecha relación con La Familia como figura retórica y la familia como institución de carácter social.

El punto "a” está bien documentado en la literatura educacional, así como también puede analizarse a partir de la segmentación de resultados SIMCE, sistema de medición de calidad a nivel nacional que se aplica a los estudiantes de cuarto y octavo año de educación básica, y a los estudiantes de segundo año de educación media y que indica que los mejores puntajes se concentran en los quintiles más ricos, mientras los puntajes más bajos se reparten en los quintiles inferiores. El punto "b" en cambio, ha sido mucho menos estudiado, aunque ya mencionado por Redondo (2005), y que tiende a justificar el éxito de los ya destinados por cuna a los buenos resultados y culpabiliza a los más pobres de su fracaso escolar; sin poner en duda el sistema y por el contrario, reproduciendo, perpetuando y legitimando el orden social y económico.

El principal eje de la "psicologización” es la práctica de la selección de los alumnos a través de los resultados obtenidos por estos en pruebas estandarizadas o "tests" de carácter particular que buscan ordenar por resultados a los postulantes a un establecimiento definido para seleccionar a aquellos con mejores resultados, reflejados en mayores puntajes, considerados los más talentosos. Los defensores de este sistema consideran que incentiva buenos resultados en todos los alumnos al propiciar la competencia por los mejores puestos en los procesos selectivos. Vale la pena hacerse algunas preguntas al respecto: ¿Cómo se selecciona a un niño o niña de 6 , 5 ó menos edad aún para entrar a un colegio? ¿Existe la "meritocracia” a esa edad? Atria (2007) se lo cuestiona de la siguiente manera: ¿son los niños agentes racionales como destinatarios de incentivos y desincentivos? El autor se responde: "los niños sólo podrán transformarse, con el tiempo, en agentes libres y responsables si hoy pueden elegir sin sufrir las consecuencias de sus malas elecciones” (Ibíd.:19).

Desde otra perspectiva, Atria (2007) considera que si el principal argumento de la descentralización es la libertad de los padres de elegir el establecimiento educacional para sus hijos/as, la selección del alumnado atentaría directamente contra esta libertad al suponer que ésta requiere la formulación de criterios que harán no elegibles a alumnos que desean entrar al establecimiento. Los defensores de la selección, según Atria, no estarían defendiendo la libertad de enseñanza sino que buscan "garantizar a los privilegiados que el privilegio del que gozan podrá ser transmitido a sus hijos” (Ibid: 117). Por tanto, esta “psicologización” reflejada en la búsqueda de buenos alumnos según resultados académicos encubre la exclusión de aquellos que no serían los adecuados para ciertos tipos de colegio protegiendo el modelo elitista a partir de la ilusión del "buen alumno", que en realidad es el estudiante de mejor situación social y cultural. 
El efecto de esta "psicologización” se observa en que se ha asumido que la forma de obtener buenos resultados escolares es a través de la ya mencionada selección de los alumnos: son ellos y sus características personales las que permitirán que los aprendizajes se produzcan, alentando poco entonces a la reflexión y mejora de los procesos pedagógicos. Los llamados "Liceos de Excelencia"² operan bajo esta lógica: solo podrán ser los mejores establecimientos si se enfocan al adiestramiento de estos alumnos que han demostrado ser los mejores, los más talentosos. La rentabilidad del liceo de excelencia es mucho mayor que la rentabilidad que ofrece un establecimiento con alumnos de bajo nivel, lo que se confirma con el modelo de la "subvención escolar preferencial".

Esta preponderancia de procesos competitivos como base de la educación genera conductas poco solidarias desde los mismos estudiantes hacia aquellos problemáticos para el sistema: estos niños y niñas deficitarios/ as provocan que se traben los caminos del modelo de aprendizaje tradicional que los niños y niñas observan y vivencian, un modelo de aprendizaje que se da como en una cinta transportadora que se basa en la tensión "alumno - atención - profesor" donde cualquiera puede transformarse en un estorbo para el avance del resto (Peña, 2009). Por otra parte, esta psicologización se ha traspasado a las estrategias de financiamiento a través de la "Subvención Escolar Diferenciada" que entrega más fondos a las escuelas a través de las matrículas de niños considerados deficitarios, bajo el entendido que ese aumento de fondos se reinvertiría en el niño, quién necesitaría mayor inversión para lograr los mismos aprendizajes que el niño no deficitario, sin tomar en cuenta la falta de evidencia que asegure que este modelo es efectivo, especialmente para estudiantes más pobres (Carnoy, 2005).

Según Flamey et. al. (2006) la ley de Subvención Escolar Preferencial (en marcha desde el año 2008) para enfrentar los problemas educativos de los establecimientos más pobres "es coherente con una lógica que privatiza las demandas sociales y los modos de resolverlas, sin abordar integralmente los factores que explican la reiterada dificultad de los establecimientos escolares para procesar y trabajar con una población diversa, y muchas veces con severos problemas socio-educativos” (Ibíd: 42). La subvención preferencial es entregada a través de subvención a la demanda, es decir, no se entrega al establecimiento por sus características generales sino por las características del alumno en particular, las que dicen relación con vulnerabilidad en términos económicos (Romaguera y Gallegos, 2010). Es una forma, a pesar de su finalidad de mejorar las condiciones de los más vulnerables, de consolidar el sistema de mercado sin querer insistir en las dificultades de carácter general que atañen a su funcionamiento.

Ahora bien, la "psicologización” no es una simple consecuencia del modelo privatizado de educación, sino que aparece a la base del modelo educativo del propio Milton Friedman, ya que éste menciona (1995: 3) que "es la inteligencia innata indudablemente lo que juega un mayor rol en determinar las oportunidades que se abrirán a los sujetos”. La competencia 
es entre y por las mejores inteligencias de los postulantes. Friedman reconoce, no obstante, que no es la única cualidad importante pero demuestra manejar la definición más clásica de inteligencia, aquella dominada por la medición de un coeficiente intelectual, a- histórica y a- teórica, que desconoce la existencia de contextos socio - culturales, sin explicitar directamente alguna referencia a aquellos alumnos que podemos suponer menos inteligentes.

Una consecuencia de esta “psicologización” del problema educativo, es la estrategia de la “medicalización”, es decir, de instalar a los niños con déficits en un modelo de diagnóstico y tratamiento por fuera de la escuela, utilizando redes estatales y privadas de profesionales del área de la salud mental como jueces fundamentales en la decisión de la continuidad del niño en el sistema escolar, perfeccionando el diseño de un problema particular e individualizado, alejado de los contextos socioculturales, un problema aún atomizado de manera extrema ya que la observación que se hace sobre el sujeto se concentra en el síntoma particular que impide el aprendizaje. La búsqueda de ese síntoma está normada en las ciencias médicas con referencias a la salud mental y la neurología, asociación que no es libre pero tampoco obvia: es así que las dificultades sociales de la pobreza terminan por convertirse en un "déficit cognitivo" o "atencional", en "hiperactividad" o en un "trastorno oposicionista - desafiante"3 , entre otras figuras diagnósticas que han sido cuestionadas principalmente por ser trastornos que parecen describir más que una enfermedad del niño, un trastorno en la relación de estos con las normas institucionales.

Este tema, ampliamente analizado a partir de las biopolíticas, tiene una estrecha relación con la constitución de las subjetividades y su forma de relación con lo público. Rose (2007) lo consigna a partir de Foucault con la obra "El nacimiento de la clínica” (1966) donde explica cómo la medicina ha ido especializándose en el cuerpo individual a través de reformas iniciadas en el Siglo XIX, cambios ontológicos, epistemológicos y técnicos que influyeron en las leyes y prácticas asistenciales, todas orientadas al "cuerpo en sí mismo”, que permanecerá hasta nuestros días como el foco de la mirada clínica. El mantenimiento de un cuerpo saludable como responsabilidad de los individuos y sus familias, en el caso de la “psicologización” pasa por un cuerpo normado que atienda en la escuela, un cuerpo dócil que se comporte de buena forma, un cuerpo infantil que se estructure en torno al cuidado amoroso de la madre, por ejemplo, dando lugar al control de grupos altamente riesgosos para el Estado, como son las mujeres y los niños/as. Según el propio Rose (Ibid.) la medicalización de los problemas sociales se basa en un agresivo imperialismo médico, basado en clamores poco realistas acerca del poder de la medicina, que termina inmiscuyéndose en los problemas morales y políticos que ciertamente no les conciernen.

Es común hablar en Chile de una “clientelización” de la Educación, es decir, de la transformación del proceso educativo en una relación de oferta y demanda poniendo el acento en el rol que le cabe al estudiante 
como cliente y los derechos y deberes que éste cumple o exige cumplir desde ese lugar particular. Esta idea de "clientelización” ha ido asociada a la creencia de que los problemas que presenta el modelo privado también son principalmente dificultades de carácter individual, a través de un aprovechamiento del estatuto de cliente, dejando de lado el importante rol que la macroestructura educativa y los contextos históricos tienen para entender las prácticas de los sujetos involucrados. Si hay una confusión entre ser alumno y cliente ésta debe ser analizada como parte de un cambio en las condiciones estructurales del modelo educativo y no exclusivamente por el rol que al parecer, de manera deliberada para sacar partido, tomarían los alumnos y sus apoderados.

Es necesario preguntarse si es que es un hecho tan ineludible que los cambios educativos han potenciado el lugar de los niños y niñas como consumidores. Debemos tomar en cuenta la posición de consumidores que detentan los niños a partir de la temprana y constante presencia de los medios de comunicación, dónde estos son un público objetivo no sólo en términos de productos que consumen como sujetos infantiles, sino también son un público en el que se trabaja la lealtad hacia la marca a futuro, especialmente en bebidas colas, cigarros y otras sustancias (Norris, 2004; Schor, 2006). Así es que nos encontramos con sujetos socializados en el consumo tempranamente, que realizan prácticas sociales asociadas a sus condiciones de clientes y consumidores, consolidando un rol más bien activo y no simples sujetos atrapados en un paradigma de la influencia (Minzi, 2002; Vergara, 2003). Los campos de la socialización de niños y niñas son múltiples, no se limitan al modelo de socialización primaria o secundaria, familia y escuela, sino que integran en un desarrollo más espiral que lineal campos sociales como la familia y la escuela, pero también medios masivos de comunicación, espacios y territorios de los colectivo (Mayall, 2002; Corsaro, 2004; James et. al., 2004). Sin embargo, en Educación la posición de cliente no es tal, ya que la decisión acerca de qué producto prefiero consumir, es del todo distinta a qué estándar de "servicio educativo" quiero optar, no solo para los estudiantes, sino que también para los padres y/o apoderados. Es decir, la "clientelización" del alumnado es un supuesto difícil de sustentar en tanto no hay una real elección en la búsqueda de escuela, especialmente cuando es la selección del alumnado/cliente la que define los estándares del establecimiento.

Ahora bien, es posible que poner la relación educativa en la lógica del servicio pudiera desconfirmar las posiciones de autoridad clásica que hay entre el profesor y su alumno, dejando ahora al primero como empleado del segundo. Es posible decir que la versión del antiguo pedagogo griego -aquel que cargaba con el peso de las tablas del aprendiz- se actualice en el presente con el profesor de algunos establecimientos donde el pago realizado por parte de los alumnos es la base de la relación institucional. Ahora bien, el profesor subordinado al alumno que paga, si bien subvierte la relación tradicional, no se aleja de la labor que un profesor realiza: trabajar para el alumno. Es la confirmación de la relación a través del dinero que lo pone a éste como un objeto de consumo que podría ser considerado 
descartable si no cumple con las necesidades planteadas por el cliente. En otras palabras, es el alumno como cliente la construcción de una nueva subjetividad, donde el alumno que asiste al establecimiento pagado tiene un poder del que carecen otros niños, dejando de lado la regla del respeto desinteresado y obediente por lo adulto, ya que ahora cuenta con un elemento que lo pone a él en un lugar preponderante de la relación.

No se debe pensar, en forma inmediata, que esto es una mala salida. No se puede olvidar que la relación profesor - alumno siempre se ha basado en el poder, y ahora ese poder se subvierte. Sin embargo, el poder del profesor que debía basarse en el saber que detentaba, coincidentemente o por causa de esto, está en franco proceso de deslegitimación. Este saber lo ponía en una posición de superioridad, no solo frente al alumno si no también frente a la comunidad de donde debía emerger este saber, sin embargo, el prestigio del profesor en Chile ha ido decayendo por múltiples razones, no siendo la subversión de la relación en el alumno cliente la principal causa. La descentralización de la educación sin la participación del gremio docente, el desmantelamiento de las facultades de educación durante la década de los ochenta, además de los cambios culturales donde el saber ya no lo detentan las instituciones consideradas tradicionalmente socializadoras por excelencia (familia y escuela) sino que es apropiado por múltiples otras instituciones, efímeras algunas; son factores fundamentales en la pérdida del prestigio tradicional del profesorado. Si ahora el alumno detenta la posición de superioridad es por el dinero-pero también- por los saberes no escolares. El temor de la escuela como institución a la apertura hacia otras fuentes de saber, como lo son los medios de comunicación y las nuevas tecnologías, podría ser un indicador de este miedo a la subversión.

Ahora bien, la desvalorización de la labor docente no debe ser entendida sólo como el resultado de una destrucción sistemática de los privilegios profesionales concretos y simbólicos que se dieron especialmente en los últimos 30 años, sino que también es importante remarcar que la carrera docente al estar relacionada con el mundo infantil y juvenil, nunca habría gozado del nivel de prestigio de otras profesiones.

El docente, mayoritariamente mujer, ocupa un rol social mal apreciado ya que se hace cargo de un grupo social poco valorado, dónde el saber disciplinar se parapeta detrás de la normatividad, de la capacidad de poner en regla al niño. La elección de la carrera docente por parte de más mujeres que hombres refleja el valor social de la profesión en nuestra sociedad: la mujer, que aún se abre espacios a nivel laboral, podría encontrar un espacio cómodo, una extensión de su labor familiar o doméstica en el mundo escolar como la madre que cuida y resguarda a los niños. El varón por su parte, prefiere mayoritariamente otras carreras donde el prestigio social es mayor, y donde, en consecuencia, la docencia no es una elección corriente. La docencia como una extensión de la vida doméstica en la "escuela guardería” coopera con la destrucción de la escuela como espacio público. Un ejemplo de esto es una política pública como la Jornada Escolar Completa (JEC) iniciada en 1997: se han alargado las horas en que los 
niños y niñas pasan en la escuela como alumnos, pero esto no ha acarreado necesariamente beneficios a nivel de la calidad de los aprendizajes (Martinic et. al., 2008), no obstante sí ha sido una política exitosa en términos de permitir a los padres de familia contar con cuidado escolar por más horas para sus hijos e hijas.

No obstante lo anterior, el niño como consumidor obtiene una posición de poder particular: puede elegir y optar por distintos objetos pero no es él quién detenta la moneda de cambio, sólo es el portador de una moneda que no es propia. La moneda en este caso es de propiedad del cuidador, ubicado generalmente en el grupo familiar. Por lo tanto, esta posición subjetiva es parte del simulacro del consumo: nuevamente el niño como alumno no es quien toma decisiones ni lleva las riendas de sus propias decisiones. Sólo el saber podría darle esa autonomía en forma legítima. Si ese saber es formal-escolar o aquel que se aprende en la calle, dependerá de las comunidades donde se inserte el sujeto y las oportunidades que ellas brinden.

Finalmente, es necesario insistir en el lugar que le cabe a la familia como institución fundamental en los procesos de descentralización y privatización. El modelo de educación descentralizada propuesto por Friedman considera que las mejoras en la calidad del sistema se deberían principalmente a la competencia, y la competencia se daría en la medida que hayan alternativas de elección para los consumidores. Es decir, en última instancia, se basa en la capacidad de los consumidores para elegir el producto o servicio que más les convenga. En este caso, como quién se educa no toma directamente la decisión, esta queda en manos de "las familias”, entendiendo por "las familias" una institución de carácter universal, es decir, presente en todos los casos educativos; y que además tiene un papel racional: podría tomar las mejores decisiones respecto de la educación de los sujetos que la componen.

Esto trae aparejados dos problemas. Uno es muy evidente y es la consecuencia de que no siempre existen familias detrás de los sujetos que se educan y lo que es más común, éstas no necesariamente toman decisiones educativas racionales: por un lado no cuentan con las herramientas necesarias (por ejemplo, ellos mismos estar educados) para tomar tal decisión; o que se vean influenciados para tomar esta decisión como pasa con cualquier producto, por una información ni veraz ni confiable (Elacqua, 2004). El primer punto es la preocupación más clásica de los investigadores en educación que han indagado en esta problemática a través de múltiples elementos que denuncian la influencia de los contextos socio culturales en el del sujeto que se educa, como el capital cultural de Pierre Bourdieu (1998) o la Sociolingüística de Bernstein (1998), entre otros autores fundamentales. El segundo punto es el que inquieta a los investigadores más pragmáticos, generando estrategias que buscan mejorar la calidad de la información que la familia tiene como insumo para decidir: más medición, más evaluación, más canales de información. Por ejemplo, la medida del “semáforo” del Ministro de Educación Joaquín Lavín ampliamente difundida el 2010 para describir escuelas con buenas mediciones nacionales para 
que los padres decidan dónde matricular a sus hijos responde a esta lógica, sin hurgar siquiera en el transfondo problemático que hemos descrito.

Otro punto menos evidente pero igualmente importante es el lugar que tiene esta supuesta renuncia del Estado en pro de las familias como herramienta ideológica. Un Estado que confía en el rol de la familia considera -finalmente- que es posible identificar esta institución como probadamente capaz de pensar y realizar lo mejor para sus miembros, y eso considerado "mejor" es coincidentemente lo mejor para el Estado 4 . Esta problemática ha sido analizada por Jacques Donzelot (1977) quien considera que la Familia se ha consolidado como un modelo de orden social, heredero de las prácticas modernas, donde tiene un rol controlador y disciplinador. Este proceso se describe como la recuperación de los niños y niñas que hicieron las clases altas, sacándolos del cuidado de los empleados y nodrizas; mientras que en los sectores bajos, el Estado le adjudicó a la familia un papel moralizador y vigilante de los vicios. La figura de la madre, en palabras de Donzelot, como fuerza centrípeta que reúne a los miembros de la familia se remarca en este modelo, garantizando un lugar social privilegiado para la mujer a través de la maternidad, cooperando en la retórica de La Madre como una santa, como mujer sacrificada, como persona buena y pura. Para aquel que quedara fuera de la familia, se crearon múltiples instituciones que cumplían el papel de cuidador: hospicios e instituciones que recogían niños de las calles, ahora bajo las condiciones de la institución y no bajo el calor familiar (Donzelot, 1977) ${ }^{5}$.

A partir de esto es posible abrir una nueva condicionante respecto de la relación Estado - Familia: en la medida que el Estado se encoge, la labor familiar crece, la maternidad se hace más acuciante, la decisión parental se vuelve con radicalidad hacia el destino del niño-hijo. Los menos favorecidos aun cuentan con un Estado cuidador, pero es un Estado cojo, carente, que se ha transformado más bien en el castigo de aquellos que no tienen el alero familiar. La escuela y el consultorio de salud municipalizados -en Chile- son el recordatorio constante de que hay que salir de ahí, que se puede salir de ahí en búsqueda de otras opciones. Cuando esta es la única opción, es reflejo de que la esfera familiar está fracasando, no ha podido llevar a cabo su labor de "hacerse cargo", como sí lo hace la familia de clase media que puede tomar a sus hijos e hijas y apoyarse por medio de un enorme tinglado de tecnologías que ordenan y solucionan sus crisis a nivel privado: psicólogos, neurólogos, pediatras, fonoaudiólogos, psicopedagogos, acupunturistas, especialistas en "flores de Bach", que se hacen cargo de volver al orden que la familia necesita pero que el Estado también requiere.

\section{A modo de cierre: Privatización de la Educación y Sujeto Político, algunos alcances}

Tanto la “psicologización”, como la “clientelización” y el énfasis en transformar a La Familia en un sujeto racional y universal, inciden en el 
desarmamiento del sujeto político al minar las bases de la constitución de lo público a través de la individuación de problemas de carácter social y de la conformación del espacio decisorio en torno a la educación dentro del ámbito de la vida privada familiar.

La falta de análisis respecto del rol público que le cabe a la educación en el modelo de educación chileno descentralizado y privatizado es decidora, especialmente cuando proviene del Estado. Este rol público dice relación con la constitución de un espacio político en el sentido clásico de la palabra, que aúna voces distintas en torno a algunos objetivos comunes que una comunidad debe plantearse para su supervivencia democrática. Esta también es una pregunta sobre el sujeto: ¿A quién se educa?, ¿a los hijos de los padres o a miembros de una comunidad más amplia? Arendt (2005) hace una precisa distinción al respecto. Para ella la educación no se explica si no es para "los nuevos", es decir, es la natalidad la causa primera de la educación y la labor de ésta es hacerse cargo del trabajo de presentarles el mundo a estos nuevos miembros de la comunidad, para hacerlos partícipes de ésta. En esto se basa también la subordinación de los alumnos a los profesores, hay algo de la tensión generacional a la base: la experiencia y la demostración de esta experiencia serían parte fundamental del trabajo adulto/docente, quién prepara al niño/a para el desarrollo de su dimensión pública dentro de un colectivo particular que es la escuela.

En el caso chileno esta situación es especialmente compleja: como una parte importante de las decisiones educativas quedan en la esfera de lo privado, podría suponerse que la privatización como tal ha tenido éxito al dejar una labor del Estado en manos de, este caso, las familias. Sin embargo, como vimos, no es posible pensar ingenuamente que las familias no están intervenidas ni menos ideologizadas por el Estado. En el caso chileno la figura retórica de la Familia se ha constituido en un dispositivo propicio para cooperar con la conservación de valores tradicionales caros a la burguesía, figura que tomaría las buenas decisiones educativas, dejando al Estado en el rol de padre de las familias pobres y poco educadas a través de algunas políticas públicas de carácter más bien asistencial. La burguesía conservadora chilena ha volcado su participación pública hacia algunos espacios muy específicos donde tiene el poder, asumiendo que en su carácter de privilegiados no deben conformar un rol público activo compitiendo con otros grupos sociales. Suele quedarse tranquila en la conformación de un remedo de espacio público que más bien es la prolongación de sus privilegios de clase, o tal vez una carga adosada a estos mismos privilegios. Arendt realiza la siguiente relación: "la moralidad cristiana, diferenciada de sus preceptos religiosos fundamentales, siempre ha insistido en que todos deben ocuparse de sus propios asuntos y que la responsabilidad política constituía una carga, en beneficio del bienestar y la salvación de quienes se liberen de la preocupación de los asuntos públicos” (Arendt, 2005: 68). Los valores tradicionales -al ser hegemónicos en Chile- no necesitan sujetos políticos activos en la construcción de nuevas ideas, menos sujetos críticos de las imperantes. El modelo de educación privada, que ensalza el derecho a la libertad no funciona si no hay familias dispuestas a luchar por 
elegir esos modelos alternativos a la escuela nacional y unificada. En rara conjunción, el modelo chileno parece decir: "Puedes educar a tus hijos donde quieras: es tu decisión. Pero esa escuela que eliges debe garantizar la entrega de algunos valores particulares que no reflejan al Estado pero que sí lo consolidan”.

Como bien lo describe Donzelot (1977), el llamado "complejo tutelar" del Estado se hace cargo directamente de los pobres, pero mantiene vigilancia ideológica sobre la familia burguesa. Ahora bien, la administración que ejerce el Estado sobre la educación chilena no es de carácter político (en su sentido público) sino que responde más bien a una administración doméstica, económica, paternalizante. Es decir, la supuesta libertad del libre mercado educativo es falsa, y el sujeto que se educa estaría siendo moralizado por parte de la visión de un Estado que tutela, y de un sistema que finalmente coopera de manera íntima con este tutelaje a través de la selección del alumnado, que tiende a normalizar a los miembros de una sociedad bajo el supuesto sello a-histórico de la inteligencia y los "resultados escolares".

No obstante, esta escuela hecha de "inteligentes" a través de la selección no reemplaza en absoluto lo que Hannah Arendt llama "elementos constitutivos de la esfera pública” (Arendt, 2005), y es más, esta tendencia a la uniformidad destruye la diferencia, la que es fundamental para la constitución de este espacio público. En otras palabras, es posible asumir que la privatización de la educación ha secuestrado la posibilidad de educar desde la esfera pública, pero también desde la política -asumiendo que ambas esferas no son siquiera equivalentes- en la medida que la privatización a través de la falsa selección meritocrática del mercado e incluyendo la supuestamente meritocrática por falaz, renuncia a la pregunta por un proyecto colectivo convirtiendo el proceso educativo en el caldo de cultivo más que para la construcción de un país, para destrucción de la vida pública y la simple protección de los derechos de los privilegiados por cuna o nivel socioeconómico.

La discusión entonces sobre la educación en Chile no debe versar tan sólo en el tema de la calidad como vector fundamental para el logro de los objetivos educativos, especialmente en el sujeto vulnerable. Cuando se concentra en el tema de la Calidad educativa la solución tiende a ser más de lo mismo: más horas, más profesores, más salas, más concentración en los ramos críticos, más información para los padres, más inclusión de estos; sin generar un debate real acerca de lo que queremos -como sociedadrespecto de la Educación de nuestros “nuevos” miembros, no sólo de nuestros hijos o hijas. El tema que parece más pertinente desde este punto de vista es reconocer y reflexionar acerca del papel que a la escuela le cabe como impulsora de la construcción de un rol público (más allá del concepto de "socialización", que a estas alturas no es una tarea que se lleve a cabo simplemente por una escuela normalizadora sin la injerencia de otras instituciones). Es la escuela y el rol que le cabe en la construcción de una democracia lo que llevaría a la discusión sobre la crisis educativa actual a 
otro nivel de mayor pertinencia, donde la los procesos de privatización podrían confrontarse con aspectos mucho más amplios que la exclusión social que propicia nuestro sistema escolar, exclusión por lo demás base de un sistema de mercado. Estos aspectos dicen relación con el proyecto social del país, con la pregunta por el sujeto político, y fundamentalmente, se dirige hacia el apuntalamiento de los derechos de quienes se educan, más allá del modelo o estilo familiar donde les tocó nacer.

Al principio dábamos cuenta de la falta de análisis entre los nexos evidentes entre política y subjetividades. Es válido preguntarse si esta falta de nexo, al menos en el ámbito educativo, dice relación finalmente con que el cambio educativo se entiende de manera insistente pero no evidente como un cambio de carácter administrativo, propio del modelo de Estado doméstico que ya hemos mencionado, donde constantemente se escamotea el sentido político y público de las decisiones del Estado post-dictatorial, que quedan como un simple proceso de reacondicionamiento burocrático llevado a cabo por expertos y no por la ciudadanía, que tiene como objetivos y valores sublimes la eficacia y la eficiencia. De ahí que las subjetividades hayan sido despejadas de este panorama y a los sujetos no les haya quedado más alternativa que instalarse en roles individualistas de carácter a-político y a-histórico. 


\section{Notas}

${ }^{1}$ Crisis que se evidencia en la calidad educativa medida a través de un sistema nacional de medición de la calidad (SIMCE) y que se debería principalmente al sistema segmentado de educación: diferentes escuelas para niños de diferentes proveniencias socio - económicas (Van der Wal, 2007).

${ }^{2}$ Son 50 Liceos de Excelencia para todo Chile que educarían a cerca de 3000 jóvenes. 30 de ellos comenzaron a funcionar el año 2011. “Queremos que los jóvenes más motivados, más esforzados, más talentosos provenientes de familias que no tienen recursos para pagar un colegio particular o no quieren poner a sus hijos en un colegio particular, tengan una vía rápida a la movilidad social. Eso son los liceos de excelencia, la vía rápida a la movilidad social”, dijo el Ministro Lavín, en www.mineduc.cl. En reportaje de Revista “Qué Pasa” del día 20 de octubre del 2011, se describe a estudiantes que entraron promedio 6 (calificación "muy buena") pero que saben un tercio de lo que se esperaba.

${ }^{3}$ Ver DSM-IV y CIE-10, manuales diagnósticos de psiquiatría, el primero de origen americano, el otro, europeo.

${ }^{4}$ En el artículo 19 de la Constitución chilena se menciona el derecho a la educación como sigue: "Los padres tienen el derecho preferente y el deber de educar a sus hijos. Corresponderá al Estado otorgar protección especial al ejercicio de este derecho”.

${ }^{5}$ Foucault hace mención de este fenómeno: “... los economistas y los médicos que estiman que el único lugar posible de remedio de la enfermedad, es el medio natural de la vida social, la familia. En ella, el costo de la enfermedad para la nación se reduce al mínimo; y desaparece también el riesgo de verla complicarse en el artificio, multiplicarse por sí misma y tomar, como en el hospital, la forma aberrante de una enfermedad de la enfermedad.” El nacimiento de la clínica (1966) Buenos Aires: Siglo XXI Editores P. 64 


\section{Bibliografía}

Arendt, H. (1989), Entre el pasado y el futuro. 8 ejercicios sobre la reflexión política. La crisis de la educación, Península, Barcelona.

Ídem (2005), La condición humana, Paidós, Barcelona.

Atria, F. (2007), Mercado y ciudadanía en educación, Flandes Indiano, Santiago de Chile.

Bernstein, B. (1993), La estructura del discurso pedagógico. Clases, códigos y control (Volumen IV), Morata, Madrid.

Bourdieu, P. (1998), La distinción. Criterio y bases sociales del gusto, Taurus, Madrid.

Corsaro, W. A. (2004), The Sociology of Childhood (Sociology for a New Century Series), Pine Forge Press, Thousand Oaks, California.

Donzelot, J. (1976), La policía de las familias, Pre-Textos, Valencia.

Flamey, L. et al. (2006), Documento de trabajo $n^{\circ} 2$ Ley subvención escolar preferencial: notas para la discusión, documento inédito Taller de Análisis sobre Proyecto de Ley Subvención Escolar Preferencial, OPECH, disponible en: www.opech.cl

Foucault, M. (1966), El nacimiento de la clínica, Siglo XXI, Buenos Aires.

Friedman, M. (1995), Public Schools: Make them private, disponible en: http://www.cato.org/pubs/briefs/bp-023.html

Elacqua G. y Fabrega R. (2004), El consumidor de la educación: El actor olvidado de la libre elección de escuelas en Chile. Programa de Promoción de la Reforma Educativa en América Latina y el Caribe (PREAL), disponile en: http:/www.opech.cl/bibliografico/Doc_Financiamiento/ consumidor_educacion.pdf

James, A. y Allison J. (2004), Constructing childhood. Theory, police and social practice, Palgrave Macmillan, Londres.

Maldonado, M. (2003), La privatización de la educación en Chile, Internacional de la Educación, Oficina regional para América Latina, disponible en: http://www.ei-ie-al.org/documentos.htm

Martinic, S., Huepe, D. y Madrid, A. (2008). “Jornada Escolar completa en Chile. Evaluación de Efectos y Conflictos en la Cultura Escolar”, en Revista Iberoamericana de Evaluación Educativa, $\mathrm{n}^{\circ} 1$, vol. 1, disponible en: http://rinace.net/riee/riee_home.html

Mayall, B. (2002), Towards a sociology for childhood: thinking from 
children's lives, Open University Press, Philadelphia.

Mizala, A., Romaguera, P. (2000), "Determinación de factores explicativos de los resultados escolares en educación media en Chile”, en Serie Economía $N^{o}$ 85, Centro de Economía Aplicada, Departamento de Ingeniería Industrial, Facultad de Ciencias Físicas y Matemáticas, Universidad de Chile.

Norris, T. (2004), "Hannah Arendt and Jean Baudrillard: pedagogy in the consumer society”, en the encyclopedia of informal education, disponible en: www.infed.org/biblio/pedagogy_consumer_society.htm

Peña, P. y Peña, M. (2007), "El saber y las tic: ¿brecha digital o brecha institucional?” en Revista iberoamericana de educación, políticas tecnológicas para la sociedad del conocimiento, $n^{\circ} 45$, OEI, Santiago de Chile Peña, M. (2011), "Hacia una Recuperación de la Subjetividad en el Proceso de Conocer en el Contexto Escolar: la pregunta por el saber en niños y niñas de educación básica chilena” en Estudios Pedagógicos, n²1, Universidad Austral, Valdivia.

Redondo, J. (2005), “El experimento chileno en educación: ¿Conduce a mayor equidad y calidad en la educación?” Última Década $n^{\circ} 22$, CIDPA Valparaíso.

Romaguera, P. y Gallegos, S. (2010), "Financiando la educación de grupos vulnerables: la subvención escolar preferencial” en Larrañaga, O. y Contreras , D. (eds.) Las Nuevas Políticas de Protección social en Chile, PNUD, Santiago de Chile (en prensa).

Rose, N. (2007), The Politics of life itself. Biomedicine, power and subjectivity in the Twenty-First century, Princeton University Press, New Jersey.

Schor, J. (2006), Nacidos para comprar. Los nuevos consumidores infantiles, Paidós, Barcelona.

Van der Wahl, Aleida (2007) "Descentralization of Education in Chile. A case of institutionalized class segregation”, tesis de maestría inédita, Leiden University, Leiden, dispoible en: http://download.ei-ie.org/docs/ IRISDocuments/EI\%20Campaigns/EFAIDS\%20Programme/2008-0001101-E.pdf

Vergara, A. (2008) "La negociación de la infancia en la relación entre padres e hijos: vinculando ciencias sociales y vida cotidiana”, en Vergara, A. y Barros, P. (eds.) Niñ@s y jóvenes en el Chile de hoy: Su lugar en los nuevos contextos familiares, Ediciones UDP-ICSO, Santiago de Chile. 